\title{
Compressive Sensing Based Image Reconstruction using Wavelet Transform
}

\author{
Sherin C Abraham ${ }^{\# 1}$, Ketki Pathak ${ }^{* 2}$, Jigna J Patel ${ }^{\# 3}$ \\ "Electronics \& Communication department, Gujarat Technological University \\ Dr. S. \& S. S. Ghandhy Government Engineering College, Surat, India \\ ${ }^{1}$ sherincheeranabraham@gmail.com \\ 3 jigna2012me@gmail.com \\ * Sarvajanik College of Engineering and Technology, Surat, India \\ ${ }^{2}$ ketki.joshi@scet.ac.in
}

\begin{abstract}
Compressive Sensing is a novel technique where reconstruction of an image can be done with less number of samples than conventional Nyquist theorem suggests. The signal will pass through sensing matrix wavelet transformation to make the signal sparser enough which is a criterion for compressive sensing. The low frequency and high frequency components of an image have different kind of information. So, these have to be processed separately in both measurements and reconstruction techniques for better image compression. The performance further can be improved by using DARC prediction method. The reconstructed image should be better in both PSNR and visual quality. In medical field, especially in MRI scanning, compressive sensing can be utilized for less scanning time.
\end{abstract}

Keyword-Compressive Sensing, Wavelet transform, Sparsity, DARC prediction

\section{INTRODUCTION}

Compressive sensing (CS) is a new compression technique where fewer samples of measurement are enough to reconstruct the image with good visual quality. The samples required are much lesser than Nyquist criterion suggests. But the non-linear reconstruction used in CS is more complex compared to linear reconstruction in conventional compression. CS will measure finite dimensional vectors. Now CS is actively researched in applications like MRI, RADAR, single pixel camera, etc. as in [1].

We consider the application in medical field. MR images are sparse in Wavelet domain. The medical images will undergo CS so that the image reconstructed will have good visual quality but with less number of measurements. MRI is slow process due to the large number of data needed to be collected while scanning a patient as in [2]. With the help of CS we can reduce the number of samples, thus reducing scan time, which will benefit patient with less radiation exposure.

In CS, there are three main principles - Sparsity, Measurements taking and Nonlinear reconstruction. The signal should be sparse - Information rate contained in the image should be much less than bandwidth - to undergo CS. If it's not sparse enough; we need to undergo the transformation of the image to make it sparse. We took wavelet transform as sparsity inducing matrix in this paper. The reconstruction of signals from lesser samples can only be possible if the chosen sparsity matrix and measurement matrix follows Restricted Isometry Property. The incoherence between these matrices is necessary for this. There are two approaches for reconstructing image at receiver side - basis pursuit and greedy algorithm. These nonlinear techniques will result in good quality reconstructed image as in [3]. In short, CS helps to reduce sampling and computation costs for sensing signals that have a sparse or compressible representation.

In paper [4], the authors introduced a novel compressive sensing based prediction measurement (CSPM) encoder. The sparse image undergoes CS by using Gaussian matrix and these measured values pass to CSPM. In CSPM, the measured matrix undergoes linear prediction and entropy encoding. Since the sparsity level of prediction residual is higher than its original image block, the performance of CS image reconstruction algorithm will be better. This CSPM encoder can achieve significant reduction in data storage and saves transmission energy. The bandwidth consumption of CSPM based CS will be considerably less which in turn increases the lifetime of sensors.

The wavelet transformed image has high frequency coefficients that are sparse and the low frequency coefficients that are not sparse. The low-frequency coefficients contain most of the energy of the image and have coherent nature. In paper [5], they measured (CS applied) the high-frequency sub band coefficients, and kept the low-frequency sub-band coefficients unchanged.

In this paper we have chosen deterministic matrices such as Hadamard matrix, random matrices such as Gaussian matrix, as measurement matrices and to attain sparsity the Daubechies wavelets are used. The nonlinear reconstruction methods used are Orthogonal Matching Pursuit (OMP) and L1 minimisation technique. The prediction methods used are Linear and DARC. 


\section{BASIC BLOCK DIAGRAM}

The signal which is K-sparse in one domain can be reconstructed from another domain which has $\mathrm{cK}$ non adaptive linear projections where $\mathrm{c}$ is small constant. The sparsity matrix and measurement matrix should be incoherent for good reconstruction as in [6].

Let $\mathrm{x}$ is a real valued, finite length, one dimensional, discrete time signal which is an $\mathrm{Nx} 1$ vector in $\mathrm{R}^{\mathrm{N}}$. When $\mathrm{x}$ is $\mathrm{K}$-sparse, i.e. $\mathrm{K}<<\mathrm{N}$, the signal $\mathrm{x}$ can undergoes $\mathrm{CS}$.

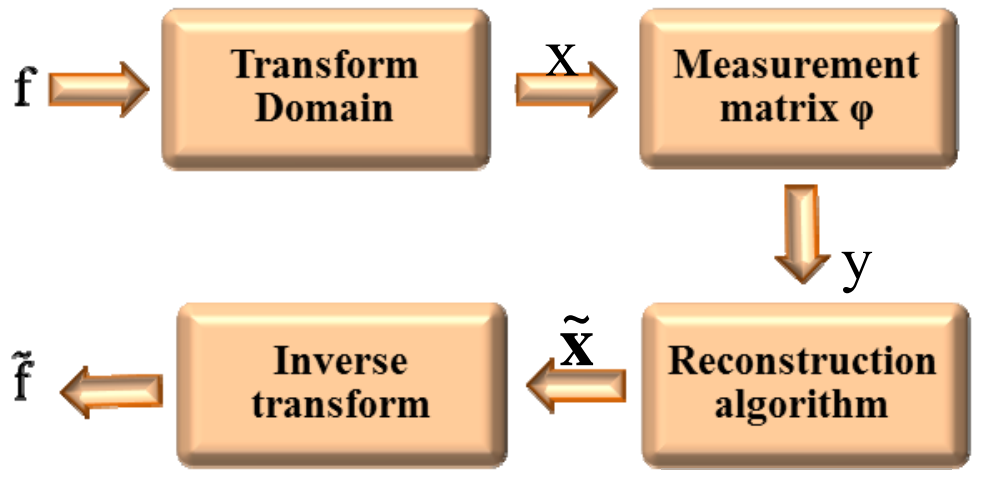

Fig. 1. Basic block diagram of compressive sensing

Thus signal $\mathrm{x}$ can be written as

$$
\mathrm{x}=\Psi \mathrm{f}
$$

where $\mathrm{f}$ is the representation of signal $\mathrm{x}$ in another domain $\Psi$.

Let $y$ be the measured vector of size $\mathrm{M} \times 1$ and $\Phi \in \mathrm{R}^{\mathrm{MxN}}$ be the measurement matrix.

$$
\mathrm{y}=\Phi \Psi \mathrm{f}=\mathrm{Acsf}=\Phi \mathrm{x}
$$

where Acs $=\Phi \Psi$ is the sensing matrix of $\mathrm{Mx} \mathrm{N}$ as in [7]

\section{III.PRoposed BLOCK DIAGRAM}

In Fig. 2, we propose a modified CSPM encoder / decoder. The prediction method and encoder is changed.

First the image will undergo 2-D wavelet transform. In paper [4], the whole wavelet coefficients are undergoing CS together and measured using a single measurement matrix. Low frequency components contain coarse information and high frequency components contain detail information. The processing of high frequency components together with low frequency components will not exploit the coherent nature of LL band and this will lead to performance degradation of the reconstructed image. So in this paper, CS will be applied separately for high frequency bands and low frequency bands as shown in Fig. 3. When applying CS to the sparse signal, we need to take measurement which is the inner product of the signal with fixed matrix. This matrix should follow the Restricted Isometry Property and incoherence. Gaussian matrix and Hadamard matrix are used as measurement matrices in this paper.

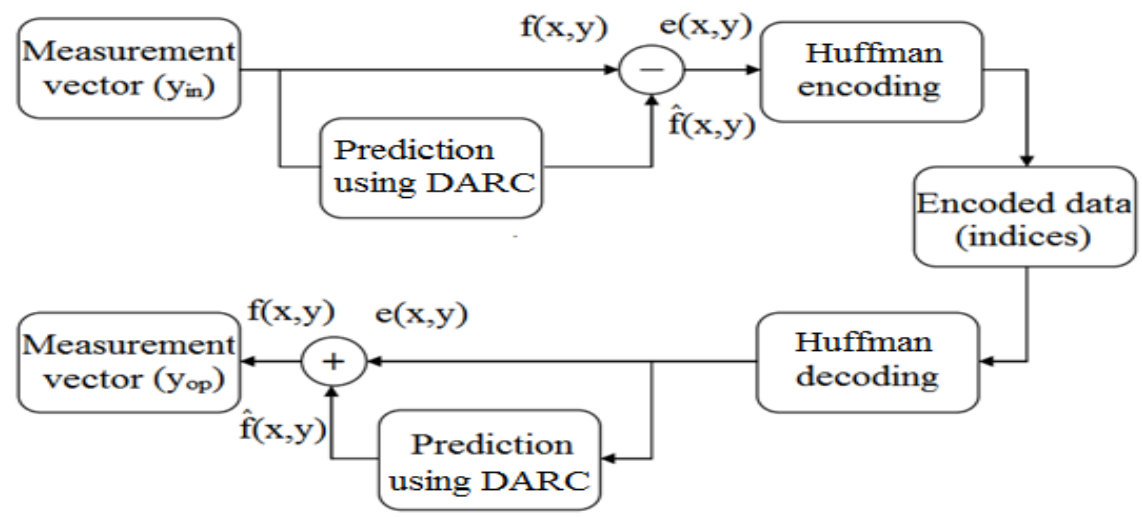

Fig. 2. Modified CSPM system

The prediction error calculated from the measured values adds more sparsity, which enhances the performance. Differential Adaptive Run Coding (DARC) prediction method is used in this paper. DARC uses weighting of three neighbouring pixels for prediction. This non-linear prediction gives better results than linear 
prediction which is used in paper [4]. DARC prediction and linear prediction are lossless prediction methods which help in reconstruction of medical images without visual degradation.

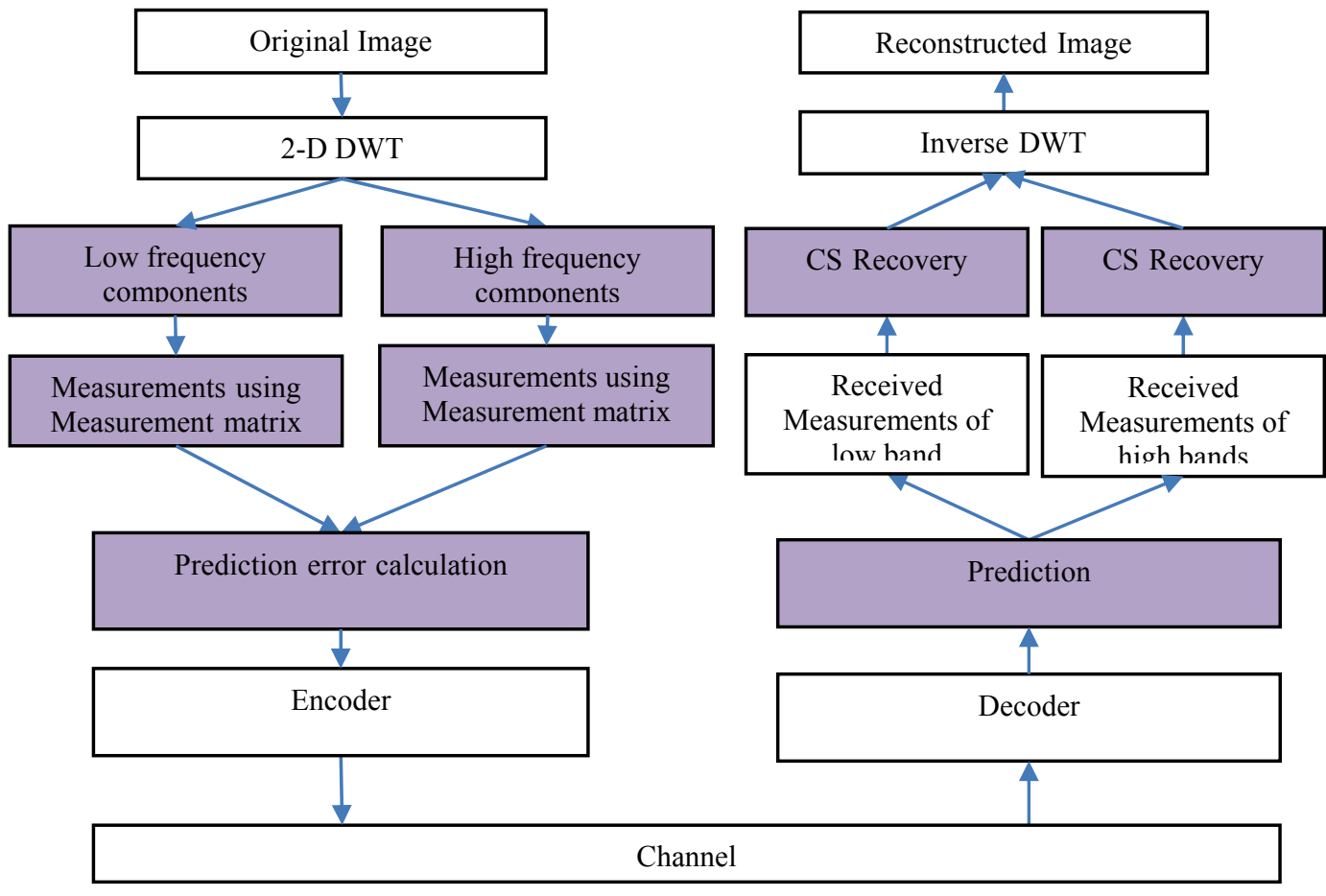

Fig. 3. Proposed block diagram

Before transmission, we need to encode the values. This will helps in the reduction of number of measurement, storage space and bandwidth. Huffman encoding and decoding which is a lossless data compression technique and having better speed in running dictionary operations is implemented in this paper.

At receiver side, we need to decode the signal received and predict the values. This is passed for reconstruction of wavelet coefficients using any basic pursuit or greedy algorithms. The OMP and L1 minimization methods are used for reconstruction in this paper. More number of iterations in OMP will give better results. After recovery, the image undergoes Inverse Wavelet Transform.

\section{IV.RESULTS}

The image should be sparse to apply CS on it. After applying wavelet transform the signal will get sparser as shown in [7]. Daubechies wavelets are also used to make image sparser. As we have already discussed the LL band and high bands are processed separately. The high bands are applied with Gaussian measurement matrix due to its random nature and LL band is applied with a different measurement matrix. The prediction error is calculated and the values are encoded by Huffman algorithm.

At the receiver side decoding and prediction are done. For CS recovery we used L1 minimisation technique for LL band and OMP for high bands which gave better results. The reconstructed images are verified using Peak Signal-to-Noise Ratio (PSNR) value which helped in evaluating the proposed algorithm.

The 256x256 size medical image is considered from [8]. Different Daubechies wavelets are used for inducing sparsity and results are shown in Table I and plotted in Fig. 4.

TABLE I Results in PSNR (dB) of Different Images with Different Daubechies Wavelets

\begin{tabular}{|c|c|c|c|c|c|}
\hline Medical images & db1 & db2 & db4 & db6 & db10 \\
\hline Image 1: Brain & 37.5 & 38.94 & 38.03 & 36.03 & 32.98 \\
\hline Image 2: Brainc1 & 41.1 & 43.23 & 40.87 & 37.94 & 33.69 \\
\hline Image 3: Brainc4 & 41.3 & 32.61 & 31.77 & 30.18 & 27.99 \\
\hline Image 4: Knee & 41.5 & 40.97 & 39.32 & 36.52 & 29.7 \\
\hline Image 5: Shoulder & 39.4 & 35.27 & 34.17 & 32.77 & 30.4 \\
\hline Image 6: MRI 1 & 36.7 & 37.19 & 33.74 & 31.12 & 27.8 \\
\hline Image 7: MRI 2 & 31.9 & 29.56 & 25.54 & 23.08 & 20.2 \\
\hline Image 8: Wrist & 42.86 & 41.7 & 40 & 39.5 & 38.12 \\
\hline
\end{tabular}




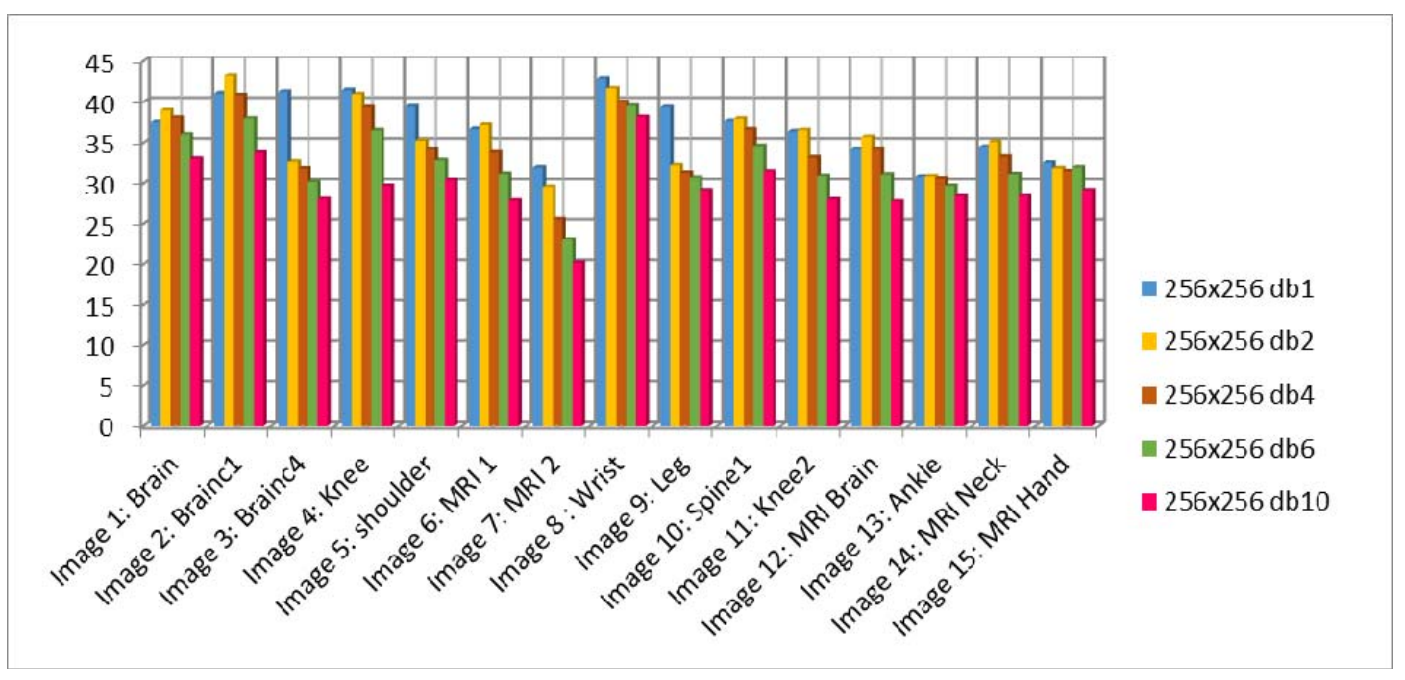

Fig. 4. Results in PSNR (dB) of different images by using different Daubechies wavelets

Haar wavelet or $\mathrm{db} 1$ wavelet is giving better results compared to other Daubechies wavelets.

The wavelet transform used is Discrete Wavelet Transform (DWT). Also the DWT wavelet transform is compared with Discrete Cosine Transform (DCT) by using Hadamard matrix as measurement matrix.

TABLE II Results in PSNR (dB) by Using DWT and DCT with Hadamard Measurement Matrix

\begin{tabular}{|c|c|c|c|c|c|c|}
\hline \multirow{2}{*}{ Medical images } & \multicolumn{3}{|c|}{ DCT } & \multicolumn{3}{c|}{ DWT } \\
\cline { 2 - 7 } & $\mathbf{1 2 8 x 1 2 8}$ & $\mathbf{2 5 6 x 2 5 6}$ & $\mathbf{5 1 2 x 5 1 2}$ & $\mathbf{1 2 8 x 1 2 8}$ & $\mathbf{2 5 6 x 2 5 6}$ & $\mathbf{5 1 2 x 5 1 2}$ \\
\hline Image 1: Brain & 32.49 & 22.53 & 21.24 & 41.4 & 37.588 & 39.14 \\
\hline Image 2: Brainc1 & 29.38 & 29.41 & 29.34 & 43.27 & 40.79 & 42.5 \\
\hline Image 3: Brainc4 & 25.29 & 22.22 & 22.08 & 39.34 & 40.14 & 40.3 \\
\hline Image 4: Knee & 27.24 & 27.3 & 27.59 & 39.89 & 36.63 & 39.3 \\
\hline Image 5: Shoulder & 29.73 & 24.58 & 22.04 & 40.03 & 41.7 & 45.9 \\
\hline Image 6: MRI 1 & 32.86 & 33.34 & 33.58 & 37.63 & 36.59 & 40.12 \\
\hline Image 7: MRI 2 & 26.92 & 26.83 & 25.72 & 29.5 & 31.51 & 31.51 \\
\hline Image 8: Wrist & 37.21 & 38.53 & 38.82 & 38.65 & 36.83 & 38 \\
\hline
\end{tabular}

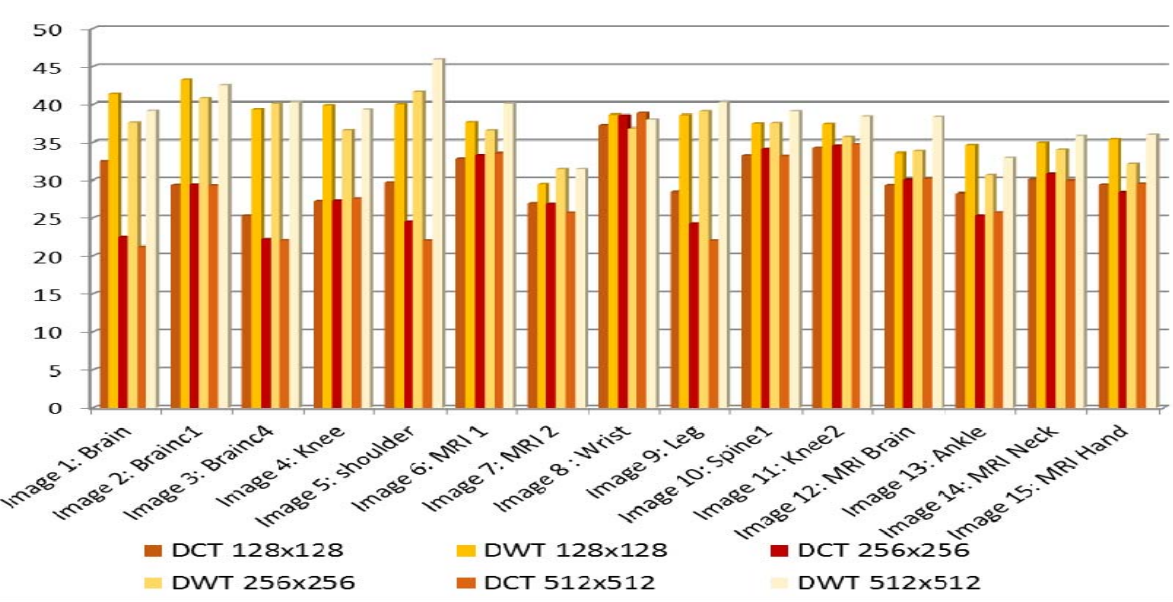

Fig. 5. Results in PSNR (dB) by using DCT and DWT with Hadamard measurement matrix 
TABLE III Comparison of Different Images by Using DCT and DWT Based on Visual Quality

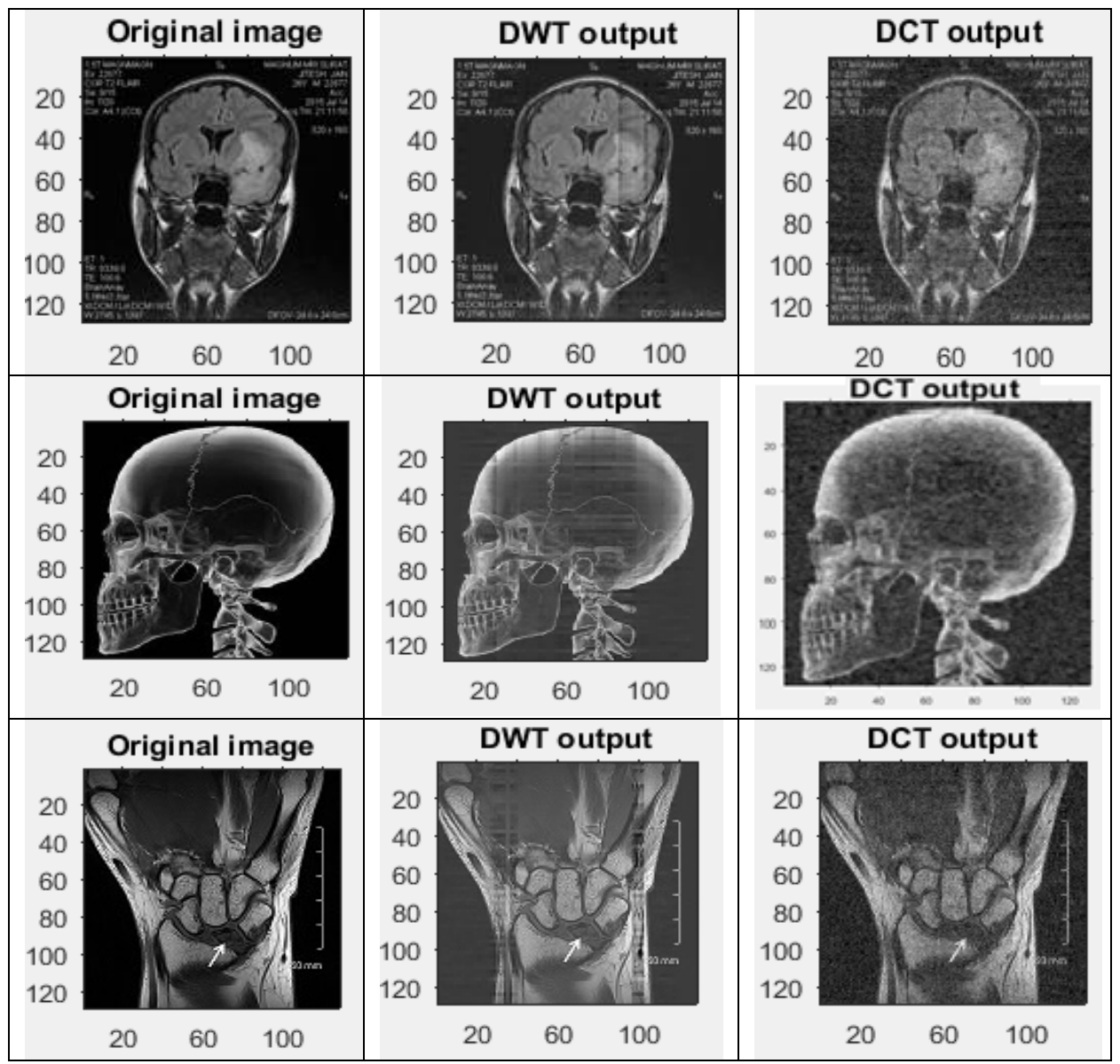

For the proposed algorithm DWT gives better results than DCT in PSNR as in Fig. 5 and better visual quality as in Table III.

The different properties of different frequency components can be exploited further when we use wavelet transform. Coherent nature of LL band can be exploited by using prediction and sparse nature of high bands can be exploited by taking random measurements. The linear prediction is also compared with DARC prediction which is also a lossless prediction technique.

TABLE IV Results in PSNR (dB) of Different Images by Using Different Prediction Techniques

\begin{tabular}{|c|c|c|c|}
\hline Medical Images & W/o prediction & Linear prediction & DARC prediction \\
\hline Image 1: Brain & 37.58 & 37.48 & 37.5 \\
\hline Image 2: Brainc1 & 40.79 & 40.68 & 41.1 \\
\hline Image 3: Brainc4 & 40.14 & 40.75 & 41.3 \\
\hline Image 4: Knee & 36.63 & 37.34 & 41.5 \\
\hline Image 5: Shoulder & 41.7 & 41.77 & 39.4 \\
\hline Image 6: MRI 1 & 36.59 & 36.41 & 36.7 \\
\hline Image 7: MRI 2 & 31.51 & 31.23 & 31.9 \\
\hline Image 8: Wrist & 36.83 & 36.81 & 42.86 \\
\hline
\end{tabular}




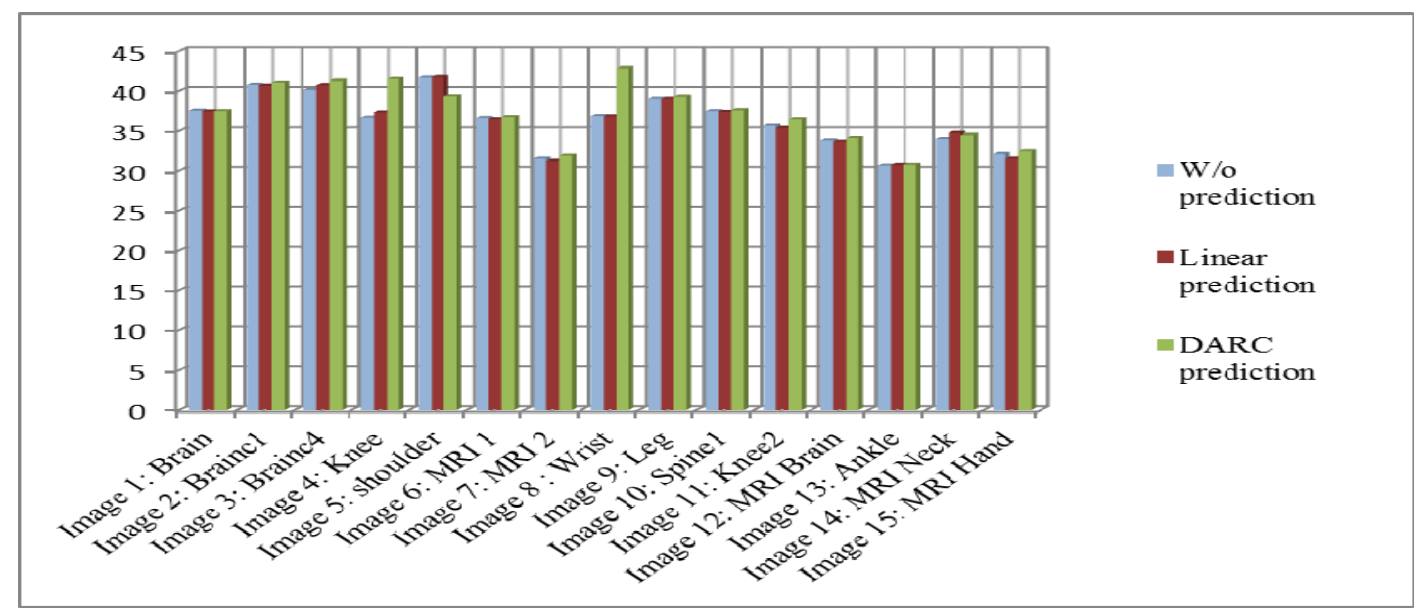

Fig. 6. Results in PSNR (dB) of different images by using different Prediction techniques

TABLE V Visual Quality of Images is Compared Between without Prediction and with DARC Prediction

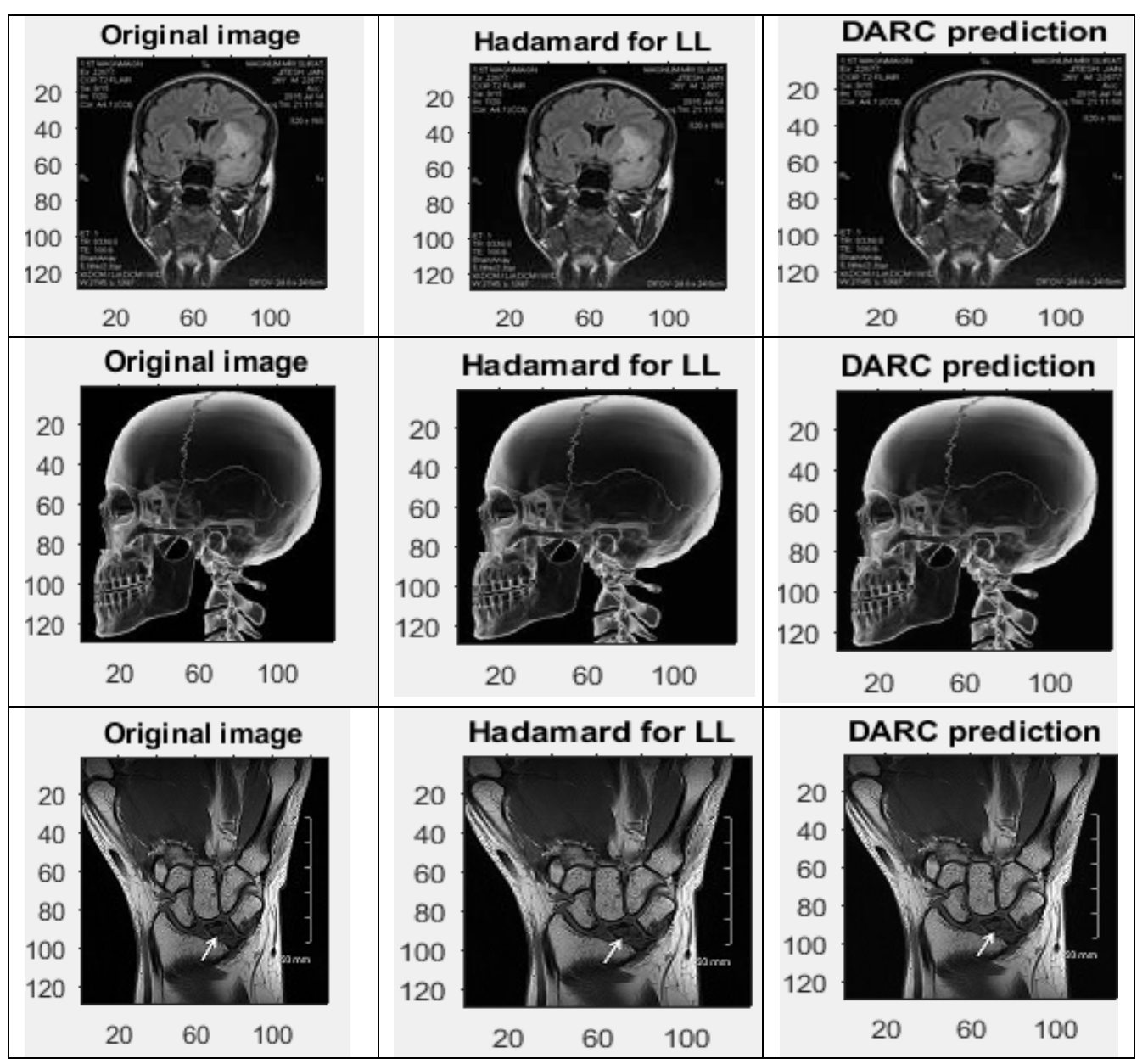

The DARC prediction will give better visual quality. More PSNR can also be achieved for an image.

The detail nature of High frequency band can be best exploited using Gaussian matrix which are pseudorandom values drawn from the standard normal distribution. The measurement matrices such as Hadamard matrix and Gaussian matrix applied for LL band. 
TABLE VI Results in PSNR (dB) of different images by using Hadamard matrix and Gaussian matrix for LL band and Gaussian matrix for high bands

\begin{tabular}{|c|c|c|}
\hline \multirow{2}{*}{ Medical images } & $\mathbf{2 5 6 x 2 5 6}$ & $\mathbf{2 5 6 x 2 5 6}$ \\
\cline { 2 - 3 } & Hadamard & Gaussian \\
\hline Image 1: Brain & 37.5 & 22.06 \\
\hline Image 2: Brainc1 & 41.1 & 19.14 \\
\hline Image 3: Brainc4 & 41.3 & 12.9 \\
\hline Image 4: Knee & 41.5 & 15.24 \\
\hline Image 5: Shoulder & 39.4 & 10.9 \\
\hline Image 6: MRI 1 & 36.7 & 12.69 \\
\hline Image 7: MRI 2 & 31.9 & 7.01 \\
\hline Image 8: Wrist & 42.86 & 24.5 \\
\hline
\end{tabular}

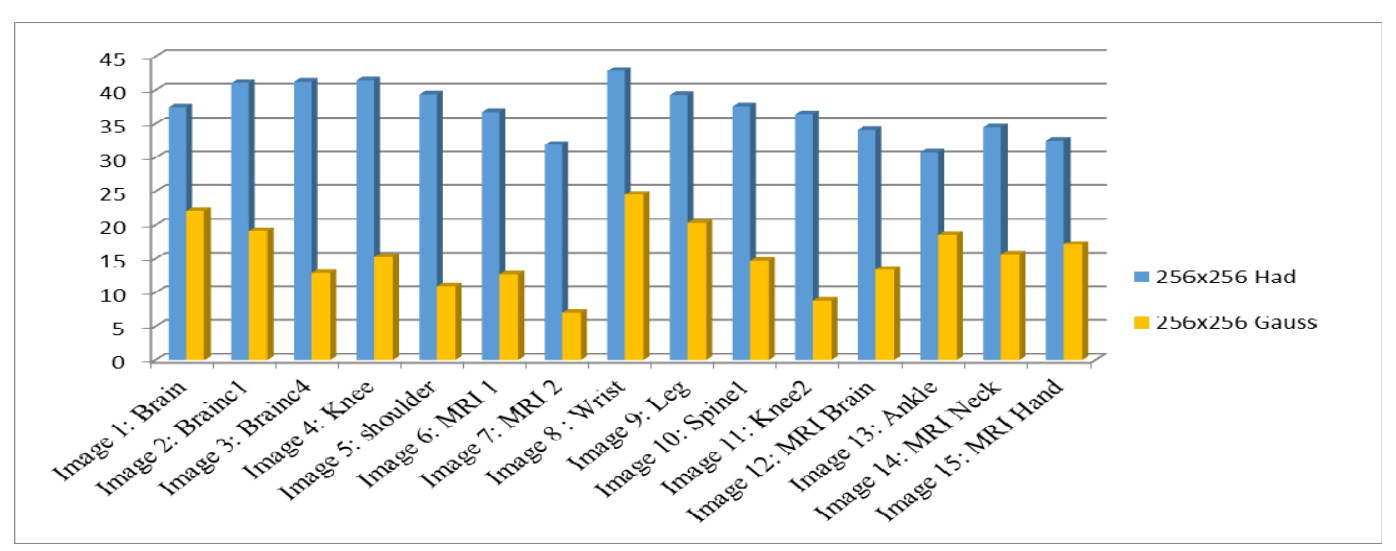

Fig. 7. Results in PSNR (dB) of Different Images by Using Different Measurement Matrix

Hadamard matrix is giving better results when used for measuring LL band. L1 minimisation reconstruction works well with these measured values.

\section{CONCLUSION}

The Compressive sensing measures small number of samples - in medical field this will help to reduce radiation time for MRI. Sparsity, incoherence and nonlinear reconstruction are three main components of CS. Wavelet transform is proven sparsity domain for many signals. The high-frequency coefficients are sparse while the low frequency coefficients are not sparse. So, both bands processed separately and used different measurement matrices and recovery algorithms. The prediction technique helps to improve the PSNR.

For LL band, Hadamard matrix is used as measurement matrix and for high bands, Gaussian matrix is used for taking measurements. L1 minimisation is used as recovery algorithm for LL band and OMP algorithm for high bands. DARC prediction method is used which give better results than linear prediction. The new proposed algorithm results in higher PSNR and better reconstructed image.

\section{REFERENCE}

[1] Saad Qaisar and et.al, "Compressive sensing: From theory to applications, a survey", IEEE Journal of communications and networks, vol.15, no.5., October 2013.

[2] Michael Lustig and et.al, Compressed sensing MRI, IEEE signal processing magazine, March, 2008.

[3] R.G. Baraniuk, Compressive Sensing [Lecture notes]. Lecture Notes in IEEE signal processing Magazine, Volume 24, issue 4, July 2007.

[4] V. Angayarkanni, S. Radha "Design of Bandwidth Efficient Compressed Sensing Based Prediction Measurement Encoder for Video Transmission in Wireless Sensor Networks", Springer Wireless Personnel Communication Journal, 2016.

[5] Xiumeni $\mathrm{Li}$ and Guoan $\mathrm{Bi}$, "Image reconstruction based on the improved compressive sensing algorithm, IEEE International Conference on Digital Signal Processing (DSP), 2015.

[6] Sherin C Abraham, Ketki Pathak, "Compressive Sensing Based Image Reconstruction - Review", International Journal of Engineering Science and Futuristic Technology, April 2017.

[7] Emmanuel J. Candès and Michael B. Wakin, An introduction to compressive sensing, IEEE Signal Processing Magazine, 2008.

[8] Image database, Civil Hospital, Surat 


\section{AUTHOR PROFILE}
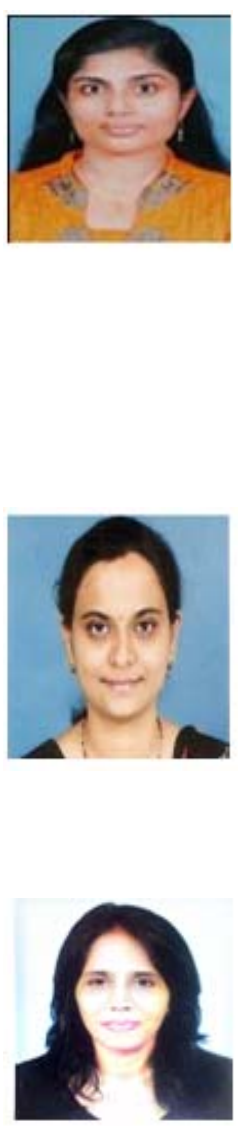

Mrs. Sherin C Abraham received Bachelor's degree in Electronics and Communication Engineering from College of Engineering, Trivandrum under Kerala University, India in 2011. She is now pursuing Master's degree in Wireless Communication and System Networks from Dr. S. \& S.S. Ghandhy Government Engineering College, under Gujarat Technological University, Surat, India. She worked as Senior Software Engineer in Robert Bosch Engineering and Business Solutions, India. She was responsible for implementation of various protocols which helps in microcontroller communication and its diagnostics. Her research area of interest includes Image Processing, Robotics, Internet Technologies and Wireless Communication.

Mrs. Ketki Pathak received Bachelor's degree in Electronics Engineering from VNSU, Surat in 2000 and Master's degree in Electronics Engineering (Communication system) from SVNIT, surat. She is pursuing Phd. Her research area of interested includes Image processing, VLSI system design, Signal Processing. She is working now as Assistant professor in Electronics and Communication department, Sarvajanik College of Engineering and Technology under Gujarat Technological University, Surat, India since 2003

Mrs. Jigna J Patel received Bachelor's degree in Computer Science from L. D. Engineering college, Ahmedabad and Master's degree in Computer Science from Sarvajanik College of Engineering and Technology, Surat under Gujarat Technological University. She is pursuing $\mathrm{PhD}$ now. Her research area of interest includes Wireless Communication and Image Processing. She is working as Assistant professor in Dr. S. \& S.S. Ghandhy Government Engineering College, under Gujarat Technological University, Surat, India. 